\title{
Sleep disordered breathing and metabolic comorbidities across sex and menopausal status in East Asians: the Nagahama Study
}

\author{
Takeshi Matsumoto ${ }^{1,2}$, Kimihiko Murase ${ }^{3}$, Yasuharu Tabara ${ }^{4}$, Takuma Minami ${ }^{1}$, \\ Osamu Kanai ${ }^{1}$, Hirofumi Takeyama ${ }^{3}$, Naomi Takahashi ${ }^{3}$, Satoshi Hamada ${ }^{5}$, \\ Kiminobu Tanizawa (1) ${ }^{1}$, Tomoko Wakamura ${ }^{6}$, Naoko Komenami ${ }^{7}$, \\ Kazuya Setoh ${ }^{4}$, Takahisa Kawaguchi ${ }^{4}$, Takanobu Tsutsumi ${ }^{4}$, Satoshi Morita ${ }^{8}$, \\ Yoshimitsu Takahashi ${ }^{9}$, Takeo Nakayama ${ }^{9}$, Toyohiro Hirai ${ }^{1}$, Fumihiko Matsuda ${ }^{4}$ \\ and Kazuo Chin ${ }^{3}$
}

@ERSpublications

Metabolic comorbidities (hypertension, diabetes, dyslipidaemia, metabolic syndrome) contribute to SDB regardless of obesity grade. We should recognise the extremely high prevalence of moderatesevere SDB in patients with obesity and metabolic comorbidities. https://bit.ly/3586f]g

Cite this article as: Matsumoto T, Murase K, Tabara Y, et al. Sleep disordered breathing and metabolic comorbidities across sex and menopausal status in East Asians: the Nagahama Study. Eur Respir J 2020; 56: 1902251 [https://doi.org/10.1183/13993003.02251-2019].

ABSTRACT It is well known that the prevalence of sleep disordered breathing (SDB) is increased in patients with obesity or metabolic comorbidities. However, the way in which the prevalence of SDB increases in relation to comorbidities according to the severity of obesity remains unclear.

This cross-sectional study evaluated 7713 community participants using nocturnal oximetry $\geqslant 2$ nights. SDB was assessed by the 3\% oxygen desaturation index corrected for sleep duration obtained by wrist actigraphy (acti-ODI3\%). SDB severity was defined by acti-ODI3\%. Obesity was defined as body mass index $\geqslant 25 \mathrm{~kg} \cdot \mathrm{m}^{-2}$.

The prevalence of SDB was 41.0\% (95\% CI 39.9-42.1\%), 46.9\% (45.8-48.0\%), 10.1\% (9.5-10.8\%) and $2.0 \%(1.7-2.3 \%)$ in normal, mild, moderate and severe SDB, respectively, with notable sex differences evident (males>post-menopausal females>premenopausal females). Comorbidities such as hypertension, diabetes and metabolic syndrome were independently associated with the prevalence of moderate-to-severe $\mathrm{SDB}$, and coincidence of any one of these with obesity was associated with a higher probability of moderate-to-severe SDB (hypertension OR 8.2, 95\% CI 6.6-10.2; diabetes OR 7.8, 95\% CI 5.6-10.9; metabolic syndrome OR 6.7, 95\% CI 5.2-8.6). Dyslipidaemia in addition to obesity was not additively associated with the prevalence of moderate-to-severe SDB. The number of antihypertensive drugs was associated with SDB ( $\mathrm{p}$ for trend $<0.001$ ). Proportion of a high cumulative percentage of sleep time with oxygen saturation measured by pulse oximetry $<90 \%$ increased, even among moderate-to-severe SDB with increases in obesity.

Metabolic comorbidities contribute to SDB regardless of the degree of obesity. We should recognise the extremely high prevalence of moderate-to-severe SDB in patients with obesity and metabolic comorbidities. 


\section{Introduction}

Obesity is one of the most important factors related to health, and its prevalence is increasing [1, 2]. Sleep disordered breathing (SDB) is a major health problem in those with obesity [3]. The prevalence of SDB has increased in comparison with past decades, and recent studies of general populations showed a prevalence of SDB as high as $60-80 \%[4,5]$. One reason for this change is assumed to be the increased rate of obesity $[6,7]$.

Although some study results have not been definitive, the associations between SDB and metabolic comorbidities such as hypertension [8], diabetes [9, 10], dyslipidaemia [11, 12] and metabolic syndrome $[13,14]$ are well known. These metabolic comorbidities were reported to be associated with high percentages of SDB in hospital- or laboratory-based studies $[13,15,16]$; however, the percentage of SDB in terms of comorbidities is not fully understood, especially in general populations. When we think in terms of cardiometabolic diseases, it is well accepted that obesity has a strong impact. In addition, obesity is a definite risk factor for SDB [3, 17]. Therefore, the association between SDB and metabolic comorbidities should be investigated considering the strong confounding factor of obesity; details of the associations according to the severity of obesity remain unclear.

The intermittent hypoxaemia caused by SDB is important in inducing systemic inflammation [18]. Recently, the hypoxic burden in patients with SDB was reported to be a factor in inducing cardiovascular diseases in addition to intermittent hypoxia [19].

Obesity, SDB and metabolic comorbidities are common conditions, and moreover have unignorable effects on public health; therefore, it is important to investigate the associations among these risk factors and SDB. Because there are significant sex differences in the prevalence of SDB $[20,21]$, investigating differences according to sex as well as menopausal status is important.

We hypothesised that obesity and metabolic comorbidities independently increase the prevalence of SDB. This study aimed to investigate the relationships among obesity, SDB and metabolic comorbidities according to sex differences and menopausal status. Using simple measurements of night-time oxygen desaturation, SDB can be underestimated because data are not based on actual sleep duration, but on the duration of measurements by oximetry. Therefore, we performed a population-based study (the Nagahama Study) and examined oxygen saturation during sleep by pulse oximetry, which was adjusted by objective sleep duration generated from actigraphy in males, premenopausal females, and post-menopausal females.

\section{Methods}

Study participants

Participants in the Nagahama study were recruited between 2013 and 2016 from the general population of Nagahama, a rural city of 125000 inhabitants located in central Japan. Community residents aged 34-80 years and living independently without any physical impairments or dysfunctions were eligible to participate. A total of 9850 individuals participated in the investigation [5]. Menopausal status was surveyed by a questionnaire and we categorised females according to premenopausal (questionnaire answer "no") and post-menopausal (questionnaire answer "yes", for whatever reasons) status. The ethics committee of Kyoto University Graduate School of Medicine approved this study, and we obtained written informed consent from all participants.

\section{Assessments of SDB}

A pulse oximeter (PULSOX-Me300; Konica Minolta, Tokyo, Japan) was put on the nondominant wrist during 4 nights of sleep. Data without oxygen-saturation signals or incomprehensible recordings were excluded from analysis. For selected data, we decided the onset and completion of sleep based on the results of actigraphy (Actiwatch 2 or the Actiwatch Spectrum Plus; Philips Respironics, Murrysville, PA,

Affiliations: ${ }^{1}$ Dept of Respiratory Medicine, Kyoto University Graduate School of Medicine, Kyoto, Japan. ${ }^{2}$ Dept of Respiratory Medicine, Saiseikai-Noe Hospital, Osaka, Japan. ${ }^{3}$ Dept of Respiratory Care and Sleep Control Medicine, Kyoto University Graduate School of Medicine, Kyoto, Japan. ${ }^{4}$ Center for Genomic Medicine, Kyoto University Graduate School of Medicine, Kyoto, Japan. ${ }^{5}$ Dept of Advanced Medicine for Respiratory Failure, Kyoto University Graduate School of Medicine, Kyoto, Japan. ${ }^{6}$ Nursing Science, Human Health Sciences, Kyoto University Graduate School of Medicine, Kyoto, Japan. ${ }^{7}$ Dept of Food and Nutrition, Kyoto Women's University, Kyoto, Japan. ${ }^{8}$ Dept of Biomedical Statistics and Bioinformatics, Kyoto University Graduate School of Medicine, Kyoto, Japan. ${ }^{9}$ Dept of Health Informatics, School of Public Health, Kyoto University Graduate School of Medicine, Kyoto, Japan.

Correspondence: Kazuo Chin, Dept of Respiratory Care and Sleep Control Medicine, Kyoto University Graduate School of Medicine, 54 Kawahara-cho, Shogoin, Sakyo-ku, Kyoto, 606-8507, Japan. E-mail. chink@ kuhp.kyoto-u.ac.jp 
USA) and used the actual sleep duration for calculations [5]. We used averaged data from a minimum of 2 days for analysis.

A $3 \%$ oxygen desaturation index (ODI) was constructed based on increments of $\geqslant 3 \%$ of drops in oxygen saturation from baseline per hour during measured sleep time by actigraphy. We used the actigraphy-modified ODI3\% (acti-ODI3\%) as an indicator of SDB that mimicked sleep apnoea, and defined the severity of SDB by acti-ODI3\% levels as follows: normal $<5$ events $\cdot \mathrm{h}^{-1}$; mild $5-<15$ events $\cdot \mathrm{h}^{-1}$; moderate $15-<30$ events $\cdot \mathrm{h}^{-1}$; and severe $\geqslant 30$ events $\cdot \mathrm{h}^{-1}$. In addition, we measured the actigraphy-modified cumulative percentage of sleep time with oxygen saturation measured by pulse oximetry $\left(S_{\mathrm{pO}_{2}}\right)<90 \%$ (acti-CT90) as a surrogate marker for continuous hypoxia, mean $S_{\mathrm{pO}_{2}}$ and minimum $S_{\mathrm{pO}_{2}}$ during sleep. In our preliminary data, acti-ODI3\% was more comparable to the apnoea/hypopnoea index (AHI) derived from attended polysomnography in 32 patients $(r=0.99, \mathrm{p}<0.001$; AHI=acti-ODI3\% $\times 1.04+1.45)$ than simply measured ODI3\% without actigraphy modification $(\mathrm{r}=0.92, \mathrm{p}<0.001$; AHI=usual ODI3\% $\times 1.27$ +2.06) (supplementary figure S1).

\section{Assessment of obesity}

We assessed three parameters of obesity: body mass index (BMI) [22], waist circumference [23] and body fat percentage [24]. BMI was calculated using the obtained height and weight data. Waist circumference was measured at the umbilical point in the standing position. Body fat percentage was calculated by bioelectrical impedance analysis.

\section{Definition of comorbidities}

Hypertension was defined as follows: ongoing treatment with antihypertensive agents or systolic blood pressure $\geqslant 140 \mathrm{mmHg}$ or diastolic blood pressure $\geqslant 90 \mathrm{mmHg}$. Data on the number of antihypertensive drugs were obtained by the following item on the questionnaire: "How many kinds of antihypertensive drugs do you take for hypertension?". Resistant hypertension was defined as systolic blood pressure $\geqslant 140 \mathrm{mmHg}$ or diastolic blood pressure $\geqslant 90 \mathrm{mmHg}$ with the use of three or more antihypertensive medications or the use of four or more antihypertensive medications regardless of blood pressure level. Otherwise, hypertension was defined as nonresistant hypertension. Diabetes was defined as follows: ongoing treatment with oral antihyperglycaemic agents and/or insulin or haemoglobin A1c $\geqslant 6.5 \%$.

The analysis of dyslipidaemia and metabolic syndrome was performed in participants in a fasting state $(\geqslant 10 \mathrm{~h})$. Dyslipidaemia was defined as follows: ongoing treatment with antihyperlipidaemic agents or low-density lipoprotein $\geqslant 140 \mathrm{mg} \cdot \mathrm{dL}^{-1}$, high-density lipoprotein $<40 \mathrm{mg} \cdot \mathrm{dL}^{-1}$ or triglycerides $\geqslant 150 \mathrm{mg} \cdot \mathrm{dL}^{-1}$. Metabolic syndrome was defined according to the Japanese Committee for the Diagnostic Criteria of Metabolic Syndrome [25], as follows: waist circumference $\geqslant 85 \mathrm{~cm}$ in males or $\geqslant 90 \mathrm{~cm}$ in females and at least two of: 1) systolic blood pressure $\geqslant 130 \mathrm{mmHg}$ or diastolic blood pressure $\geqslant 85 \mathrm{mmHg}$ or the use of antihypertensive drugs; 2) fasting plasma glucose $\geqslant 110 \mathrm{mg} \cdot \mathrm{dL}^{-1}$ or the use of drugs for diabetes; and 3) high-density lipoprotein $<40 \mathrm{mg} \cdot \mathrm{dL}^{-1}$ or triglycerides $\geqslant 150 \mathrm{mg} \cdot \mathrm{dL}^{-1}$ or the use of antihyperlipidaemic drugs.

\section{Statistics}

Values are expressed as mean \pm sD, frequency (95\% confidence interval) or median (interquartile range). Multiple logistic regression analyses were performed to assess the relationships among SDB and obesity parameters and/or comorbidities. The models were adjusted by age, sex, alcohol status and smoking status. The Cochran-Armitage test was used as a trend test. A two-tailed p-value $<0.05$ was deemed statistically significant. We conducted statistical analyses using JMP Pro 13.0.0 (SAS Institute, Cary, NC, USA).

\section{Results}

\section{Study participants}

Supplementary figure S2 is a flowchart of study enrolment. The total sample for the analysis was comprised of 7713 individuals from the 9850 initial participants. Table 1 summarises the clinical characteristics of study participants. The differences between included and not-included participants were as follows: age $(57.9 \pm 12.1$ years versus $58.5 \pm 13.5$ years, $\mathrm{p}=0.03)$, sex $(32.5 \% / 23.4 \% / 44.2 \%$ versus $33.7 \%$ / $23.6 \% / 42.8 \%$ in males/premenopausal females/post-menopausal females, respectively, $\mathrm{p}=0.40$ ) and BMI $\left(22.3 \pm 3.3 \mathrm{~kg} \cdot \mathrm{m}^{-2}\right.$ versus $\left.22.2 \pm 3.4 \mathrm{~kg} \cdot \mathrm{m}^{-2}, \mathrm{p}=0.59\right)$. Mean BMI was in descending order: males>post-menopausal females>premenopausal females. A similar tendency was seen in waist circumference, while the order of body fat percentage was post-menopausal females $>$ premenopausal females $>$ males. As to complications, males had the highest percentages of hypertension, diabetes and metabolic syndrome, while post-menopausal females had the highest percentage of dyslipidaemia. The associations among obesity parameters are shown for males, premenopausal females and post-menopausal females in supplementary table S1. As shown in supplementary table S1, significant correlations were seen 
TABLE 1 Baseline characteristics of study participants

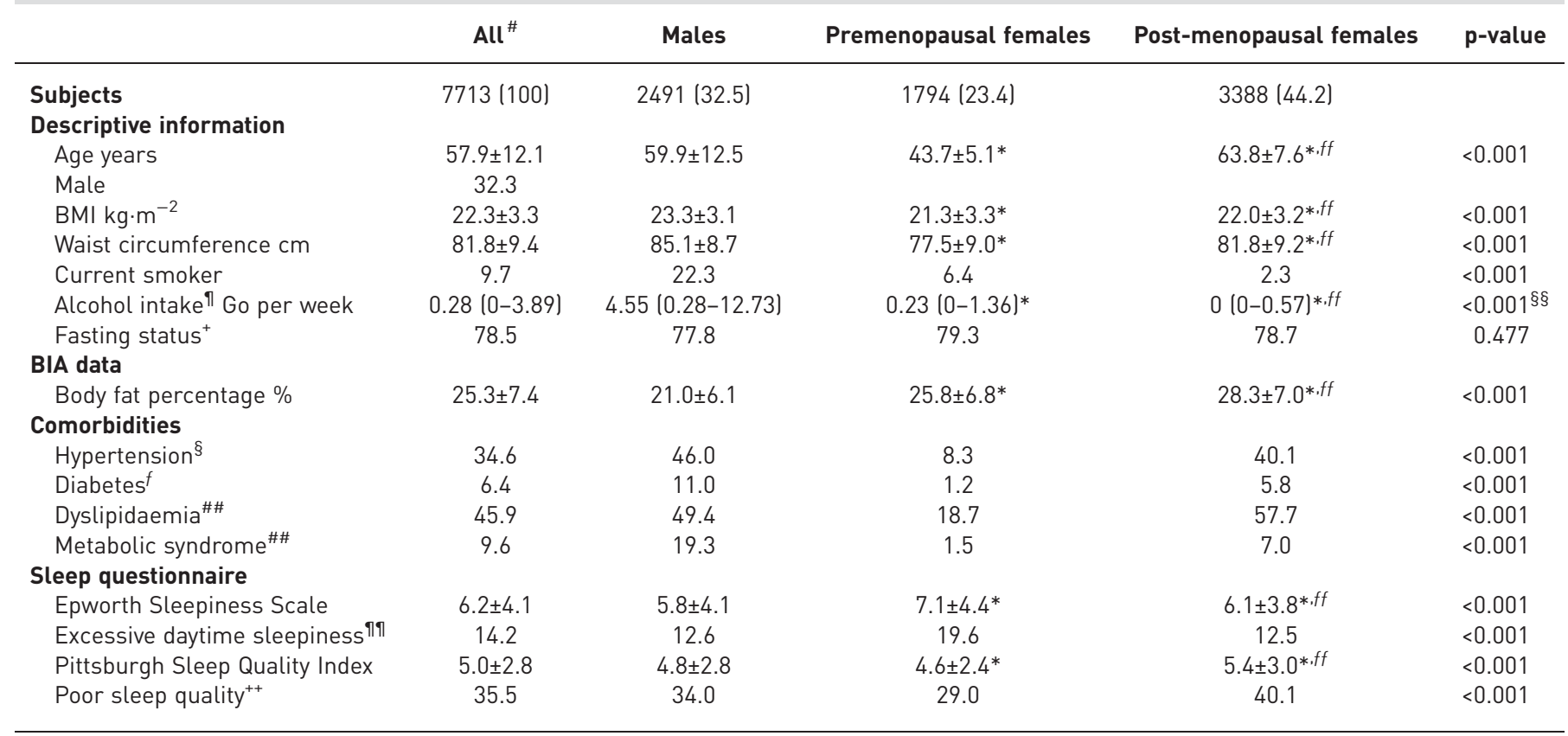

Data are presented as $\mathrm{n}(\%)$, mean $\pm \mathrm{SD}, \%$ or median (interquartile range), unless otherwise stated. Continuous variables were calculated by ANOVA, unless otherwise stated. BMI: body mass index; Go: Japanese traditional units of alcohol (1 Go corresponds to $22 \mathrm{~g}$ ethanol); BIA: bioelectrical impedance analysis. \#: data on menopause were unavailable for $\mathrm{n}=40$ females; data on hypertension, diabetes, dyslipidaemia and metabolic

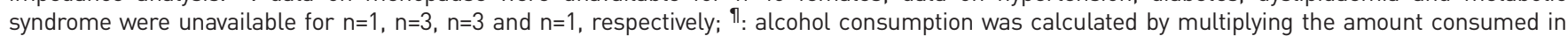
a single day and number of drinking days per week; ${ }^{+}$: last meal $\geqslant 10 \mathrm{~h}$ prior; ${ }^{\S}$ : hypertension was considered present when the patient was treated for systemic hypertension or systolic blood pressure $\geqslant 140 \mathrm{mmHg}$ or diastolic blood pressure $\geqslant 90 \mathrm{mmHg} ;{ }^{f}$ : diabetes was considered present when the patient was treated for diabetes or haemoglobin A1c $\geqslant 6.5 \%$; \#\#: data were analysed for 6051 participants: 1937 males, 1422 premenopausal females, and 2665 post-menopausal females (data on menopause were unavailable for $n=27$ females) in fasting status; ११: those with an Epworth Sleepiness Score $>10$ were defined as having excessive daytime sleepiness; ${ }^{++}$: those with a Pittsburgh Sleep Quality Index $\geqslant 6$ were defined as having poor sleep quality; ${ }^{\S \S}$ : Kruskal-Wallis test. *: $p<0.05$ versus males; ${ }^{f f}$ : $p<0.05$ versus premenopausal females.

among BMI and waist circumference, waist circumference and body fat percentage, and BMI and body fat percentage.

\section{SDB prevalence}

SDB parameters in males $(n=2491)$ and females according to menopausal status $(n=1794$ premenopausal females, $n=3388$ post-menopausal females) are shown in table 2 . The prevalence of SDB was $41.0 \%(95 \%$ CI 39.9-42.1\%), 46.9\% (45.8-48.0\%), 10.1\% (9.5-10.8\%) and 2.0\% (1.7-2.3\%) in normal, mild, moderate and severe SDB, respectively. There was a notable sex difference in prevalence: males>post-menopausal females>premenopausal females. The prevalence of SDB in East Asians was not very different from that reported in studies of Caucasian or Hispanic/Latino participants [4, 26, 27] (supplementary figure S3). For the sensitivity analysis, we included continuous positive airway pressure (CPAP) users $(n=41)$ into the moderate-to-severe SDB group and then calculated the prevalence of SDB. The prevalence of SDB then changed to be $40.8 \%$ (95\% CI $39.7-41.9 \%), 46.6 \%(45.5-47.8 \%)$ and $12.6 \%(11.9-13.3 \%)$ in normal, mild and moderate-to-severe SDB, respectively. A slight increase in the prevalence of moderate-to-severe SDB was then noted.

\section{Association between obesity parameters and SDB}

The prevalence of moderate-to-severe SDB according to age and BMI categories is shown in figure 1 . Increased age and BMI were both related to a greater presence of moderate-to-severe SDB. Those aged $<40$ years were much more susceptible to SDB when they were obese (BMI $\geqslant 25 \mathrm{~kg} \cdot \mathrm{m}^{-2}$; unadjusted OR 40.6, 95\% CI 14.9-110.5), compared to nonobese patients. When the impact of specific obesity parameters was investigated, a significant association was seen for each obesity parameter (supplementary table S2). Given the values of point estimation of odds ratio, in all obesity parameters, the association between the obesity parameter and SDB was the strongest in premenopausal females, followed by males and post-menopausal females. 
TABLE 2 Sleep disordered breathing (SDB) parameters in males and females according to menopausal status

\begin{tabular}{|c|c|c|c|c|c|}
\hline & All & Males & $\begin{array}{c}\text { Premenopausal } \\
\text { females }\end{array}$ & $\begin{array}{l}\text { Post-menopausal } \\
\text { females }\end{array}$ & p-values \\
\hline Subjects & $7713(100)$ & 2491 (32.5) & $1794(23.4)$ & $3388(44.2)$ & \\
\hline Normal & $41.0(39.9-42.1)$ & $19.0(17.5-20.6)$ & $73.6(71.5-75.6)$ & $39.8(38.2-41.4)$ & $<0.001$ \\
\hline Mild & $46.9(45.8-48.0)$ & $57.5(55.6-59.5)$ & $24.8(22.9-26.9)$ & $50.8(49.1-52.5)$ & \\
\hline Moderate & $10.1(9.5-10.8)$ & $18.9(17.4-20.5)$ & $1.6(1.1-2.2)$ & $8.2(7.4-9.2)$ & \\
\hline Actigraphy-modified CT90 \% & $0.18(0.05-0.75)$ & $0.44(0.11-1.54)$ & $0.05(0.02-0.15)^{*}$ & $0.19(0.06-0.69)^{*, \#}$ & $<0.001$ \\
\hline Mean $\mathrm{S}_{\mathrm{pO}_{2}} \%$ & $96.8(95.9-97.6)$ & $96.2(95.3-96.9)$ & $97.9(97.3-98.4)^{*}$ & $96.7(95.8-97.3)^{*, \#}$ & $<0.001$ \\
\hline Minimum $\mathrm{S}_{\mathrm{po}_{2}} \%$ & $84.8(81.2-87.6)$ & $83.7(79.4-86.7)$ & $86.4(83.3-88.9)^{*}$ & $84.7(81.4-87.3)^{*, \#}$ & $<0.001$ \\
\hline
\end{tabular}

Data are presented as $\mathrm{n}(\%)$ or median (interquartile range), unless otherwise stated. Continuous variables were calculated using the KruskalWallis test. Total sleep duration (from sleep onset time to wake-up time) by actigraphy and actual sleep duration (sleep duration after exclusion of wake-time after sleep onset from total sleep duration) were determined using the standard factory-default algorithm. The $3 \%$ oxygen desaturation index (ODI3\%) adjusted for actual sleep duration was used as an indicator of SDB, and the severity of SDB was defined by ODI3\% levels as follows: normal $<5$ events $\cdot h^{-1}$, mild $5-<15$ events $\cdot h^{-1}$, moderate $15-<30$ events $\cdot h^{-1}$ and severe $\geqslant 30$ events $\cdot h^{-1}$. CT90: cumulative percentage of sleep time with oxygen saturation measured by pulse oximetry $\left(S_{\mathrm{pO}_{2}}\right)<90 \%$. *: p<0.05 versus males; * $<0.05$ versus premenopausal females.

\section{Association between comorbidities and SDB according to severity of obesity}

Prevalence of moderate-to-severe SDB according to BMI and the presence of hypertension or diabetes in the whole cohort, and in male, premenopausal female and post-menopausal female subgroups is shown in figure 2. In the whole cohort and all subgroups, moderate-to-severe SDB was found in those with comorbidities, and the presence of diabetes rather than hypertension was more closely related to the

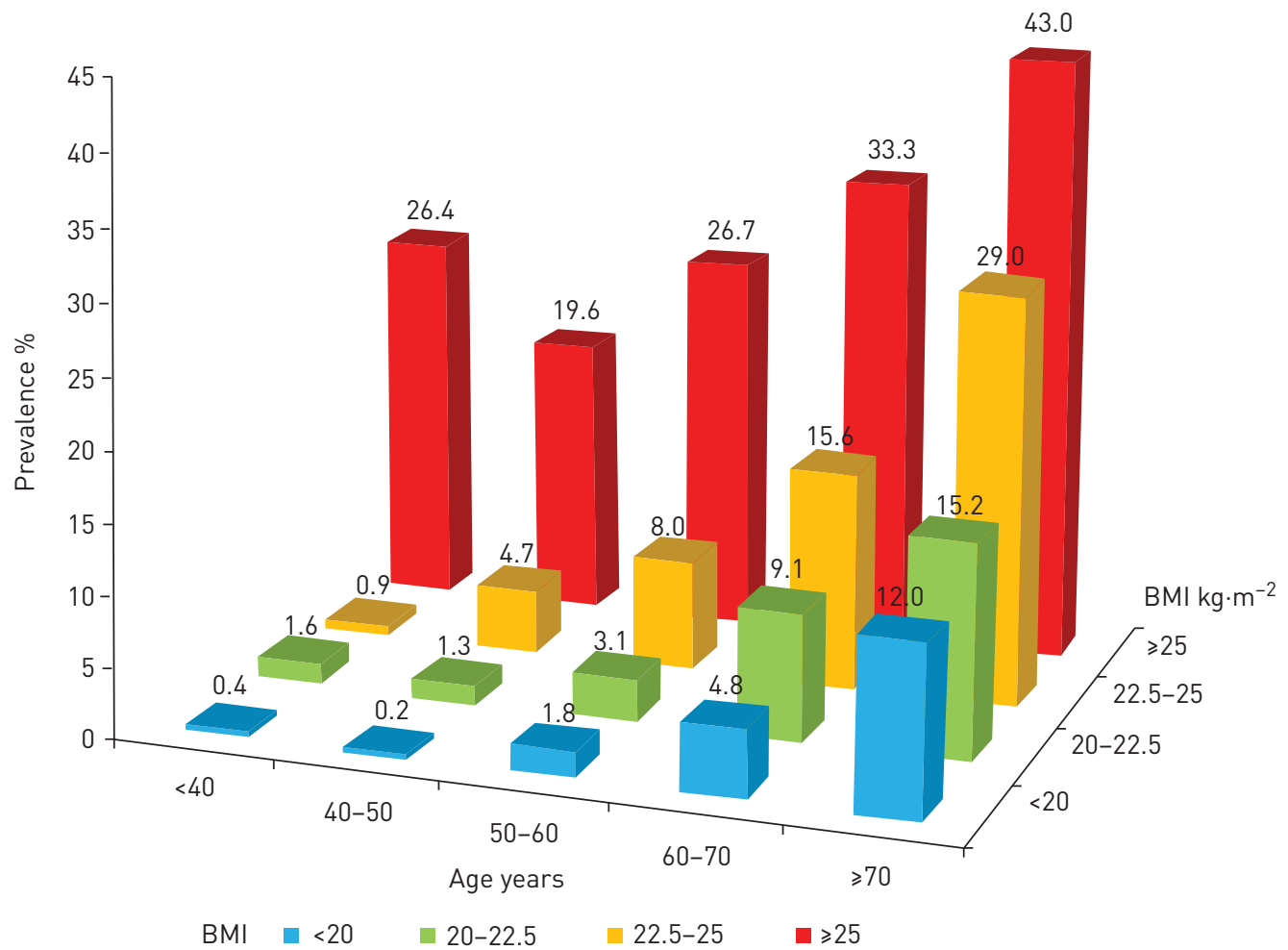

FIGURE 1 Prevalence of moderate-to-severe sleep disordered breathing (SDB) according to age and body mass index (BMI) groupings. BMI is categorised from $<20 \mathrm{~kg} \cdot \mathrm{m}^{-2}$ to $\geqslant 25 \mathrm{~kg} \cdot \mathrm{m}^{-2}$ in units of 2.5 and age is categorised from $<40$ years to $\geqslant 70$ years in units of 10 . The number above each bar graph represents the raw estimate of the percentage of moderate-to-severe SDB. Moderate-to-severe SDB is defined by $\geqslant 15$ events $\cdot \mathrm{h}^{-1}$ of the $3 \%$ oxygen desaturation index modified by actigraphy. 

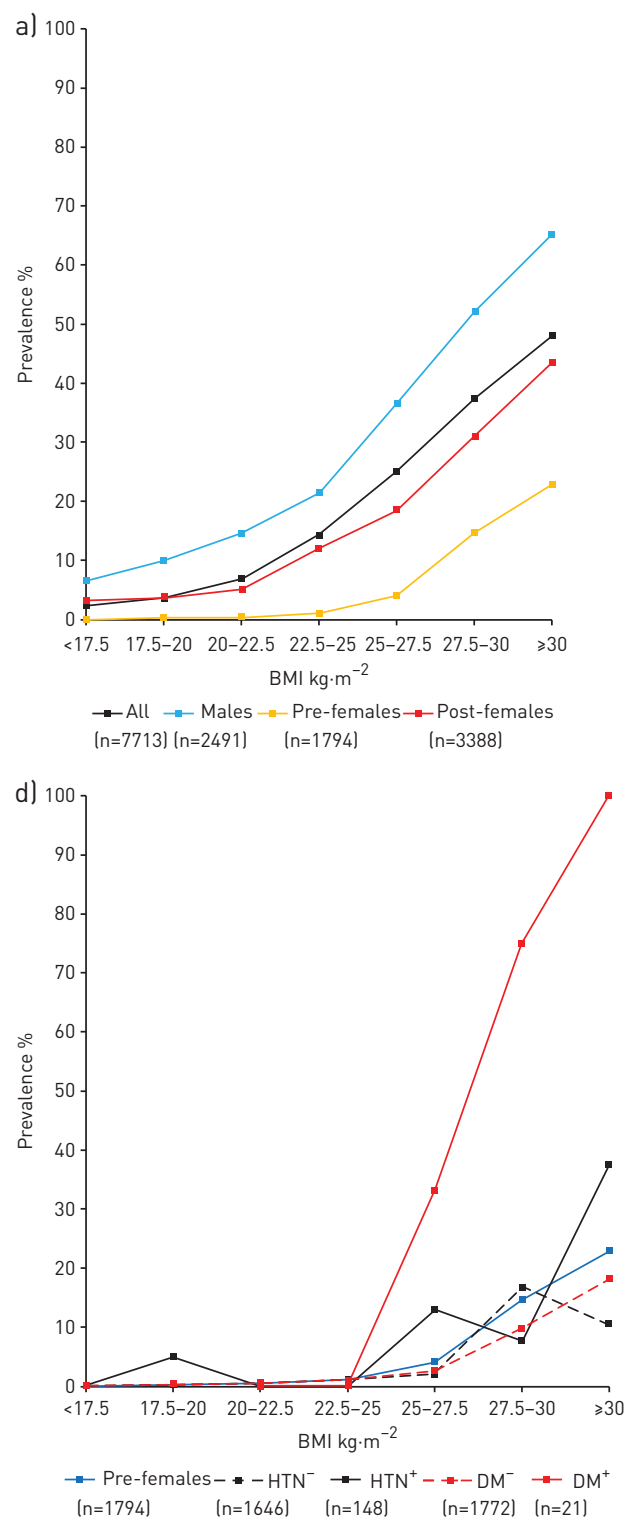
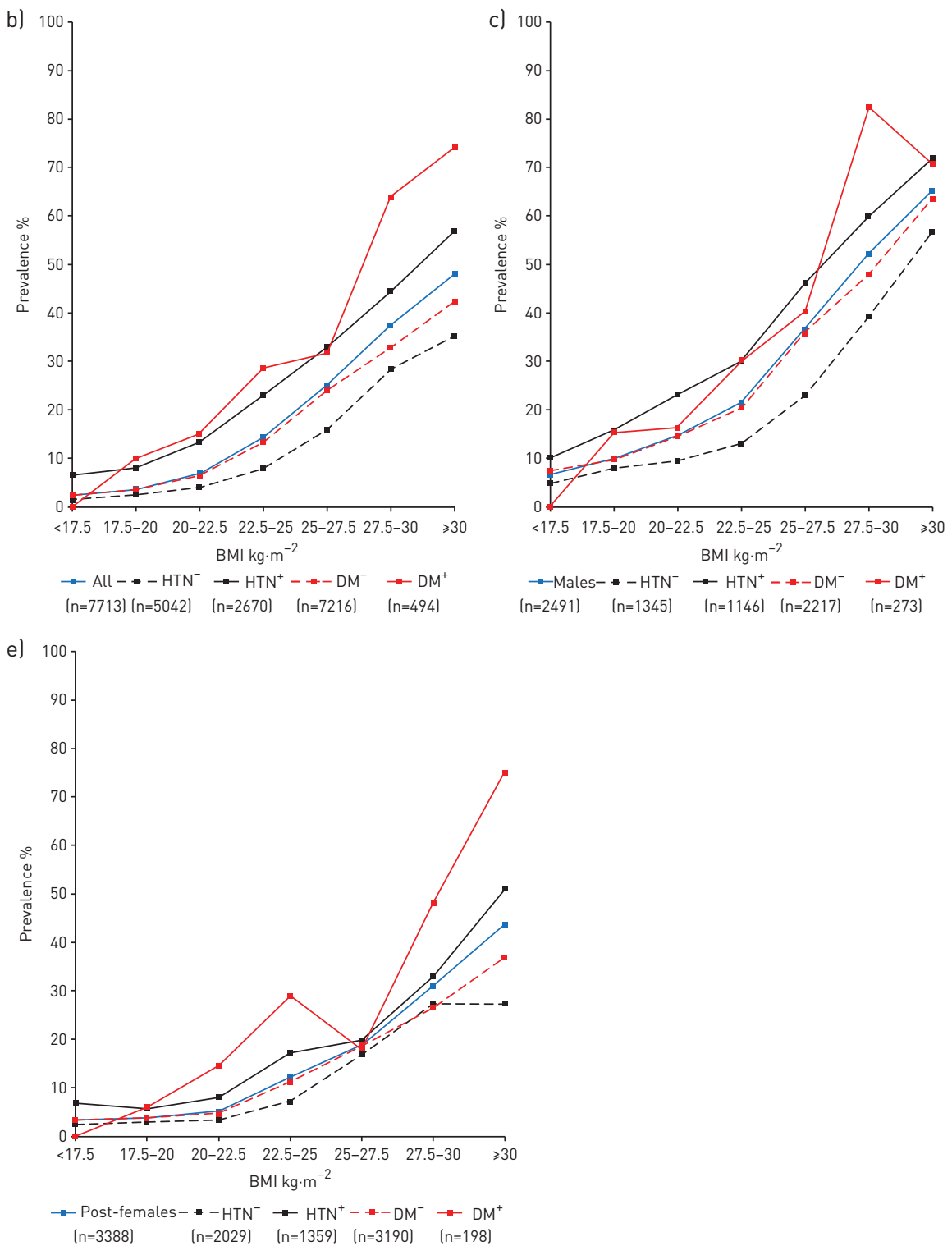

FIGURE 2 Prevalence of moderate-to-severe sleep disordered breathing (SDB) in males and females according to menopausal status in relation to hypertension and/or diabetes. a) Total population, males and females according to menopausal status; b) presence or absence of hypertension/ diabetes in all participants; c) presence or absence of hypertension/diabetes in males; d) presence or absence of hypertension/diabetes in premenopausal females; e) presence or absence of hypertension/diabetes in post-menopausal females. Data on dyslipidaemia and metabolic syndrome were limited to participants with a fasting state. HTN: hypertension; DM: diabetes; Pre-females: premenopausal females; Post-females: post-menopausal females.

presence of moderate-to-severe SDB. In particular, premenopausal females with diabetes had the highest prevalence of moderate-to-severe SDB. For hypertension, when analysed by the number of antihypertensive drugs, the prevalence of moderate-to-severe SDB was significantly increased according to the number of drugs used regardless of blood pressure level ( $\mathrm{p}$ for trend $<0.001$ ) (figure $3 \mathrm{a}$ ). In addition, although resistant hypertension was present in a small number of patients $(n=110)$, it was significantly associated with a high prevalence of moderate-to-severe SDB (figure 3b). When obesity was defined as $\mathrm{BMI} \geqslant 25 \mathrm{~kg} \cdot \mathrm{m}^{-2}$, coincidence of both obesity and any comorbidity with the exception of dyslipidaemia, compared to a single factor, resulted in a high prevalence of moderate-to-severe SDB (figure 4). After adjusting for covariates, coincidence of both obesity and comorbidities resulted in a high prevalence of moderate-to-severe SDB (OR 8.2, 95\% CI 6.6-10.2 in hypertension; OR 7.8, 95\% CI 5.6-10.9 in diabetes; OR 5.8, 95\% CI 4.6-7.4 in dyslipidaemia; OR 6.7, 95\% CI 5.2-8.6 in metabolic syndrome). These odds ratios were compared with those of participants with neither hypertension nor obesity, with neither diabetes nor obesity, with neither dyslipidaemia nor obesity, and with neither metabolic syndrome nor 
a)

50
45

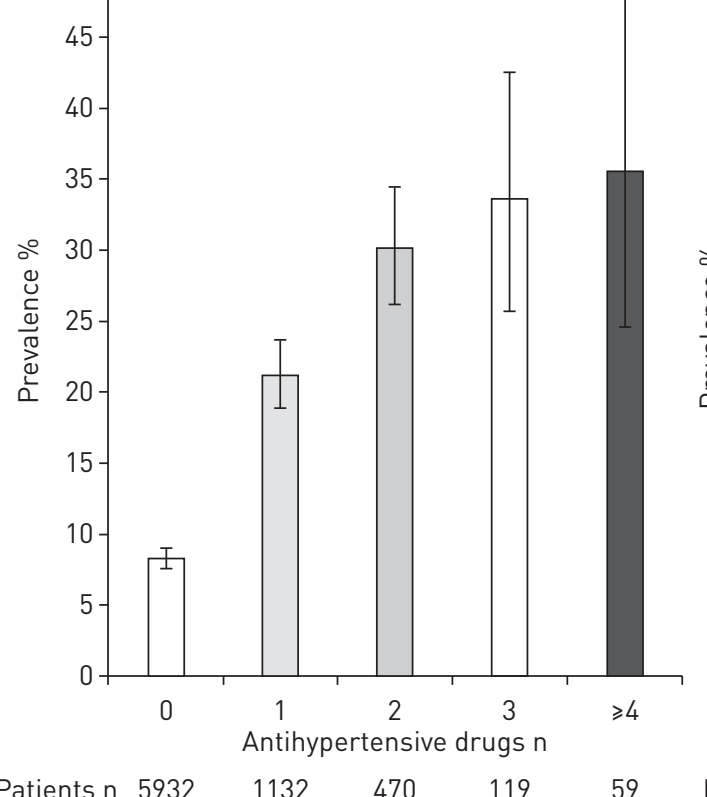

Patients n 5932

1132

470

119

59

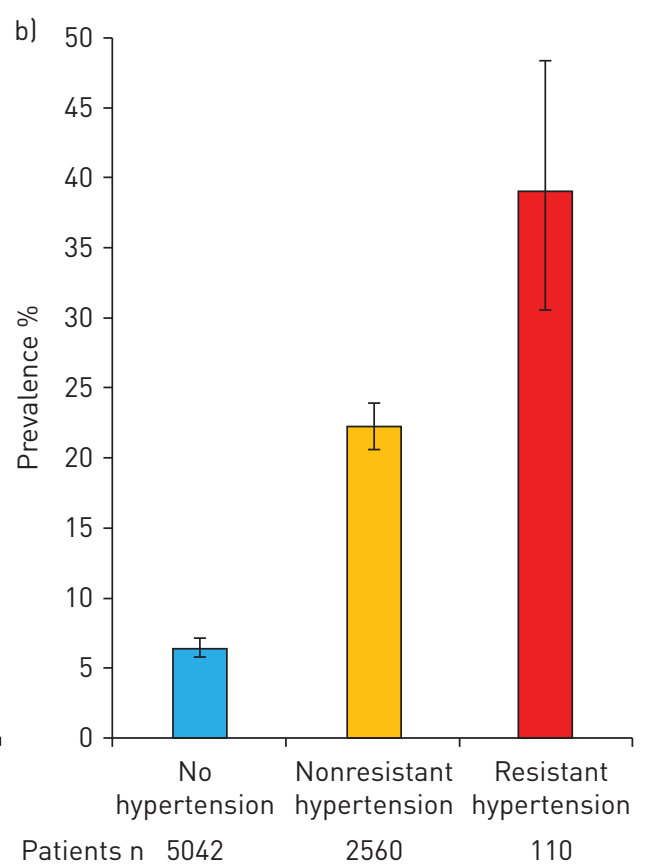

FIGURE 3 Prevalence of moderate-to-severe sleep disordered breathing (SDB) according to the number of antihypertensive drugs and the presence or absence of resistant hypertension. al Prevalence of moderate-to-severe SDB according to the number of antihypertensive drugs; b) prevalence of moderate-to-severe SDB according to the presence or absence of resistant hypertension. Data are presented as point estimates $(95 \% \mathrm{Cl})$. Hypertension is considered present according to ongoing pharmacological treatment with antihypertensive drugs or systolic blood pressure $\geqslant 140 \mathrm{mmHg}$ or diastolic blood pressure $\geqslant 90 \mathrm{mmHg}$. Resistant hypertension is defined as systolic blood pressure $\geqslant 140 \mathrm{mmHg}$ or diastolic blood pressure $\geqslant 90 \mathrm{mmHg}$ with the use of three or more antihypertensive medications or use of four or more antihypertensive medications regardless of blood pressure level. Otherwise, hypertension is defined as nonresistant hypertension.

obesity, respectively. Although obesity was a strong factor for the presence of moderate-to-severe SDB, the presence of comorbidities was also an independent factor for the presence of moderate-to-severe SDB, even in the absence of obesity (OR 2.3, 95\% CI 1.8-2.8 in hypertension; OR 1.5, 95\% CI 1.1-2.1 in diabetes; OR 1.5, 95\% CI 1.2-1.9 in dyslipidaemia; OR 2.2, 95\% CI 1.6-3.0 in metabolic syndrome). However, dyslipidaemia in addition to obesity was not additively associated with SDB (figure 5). For sensitivity analyses, we changed the cut-off level of SDB to acti-ODI3\% 5 events $\cdot \mathrm{h}^{-1}$ or 20 events $\cdot \mathrm{h}^{-1}$ and show the percentages and odd ratios in supplementary figures S4-S7. The results were similar to the original analyses in that the impact was higher in the order of doubly negative, only comorbidity present, only obesity present, and doubly positive, and dyslipidaemia in addition to obesity was not additively associated with SDB.

\section{Association between SDB and clinical symptoms}

Excessive daytime sleepiness (EDS) was not related to the presence of moderate-to-severe SDB (EDS $11.7 \%$ (95\% CI 9.9-13.7\%) versus no EDS $12.2 \%$ (95\% CI 11.4-13.0\%); $\mathrm{p}=0.65$ ). When the effect of the combination of metabolic comorbidities or obesity with EDS was evaluated, metabolic comorbidities or obesity rather than EDS was associated with a higher prevalence of SDB (supplementary figure S8). In addition, poor sleep quality was not related to the presence of moderate-to-severe SDB (poor sleep quality $13.0 \%$ (95\% CI $11.8-14.4 \%$ ) versus no poor sleep quality $11.6 \%$ (95\% CI $10.7-12.5 \%)$; $\mathrm{p}=0.61$ ), and metabolic comorbidities or obesity rather than poor sleep quality was associated with a higher prevalence of SDB (supplementary figure S9).

\section{Association between acti-ODI3\% and acti-CT90 according to severity of obesity}

The prevalence of high acti-CT90 among participants with moderate-to-severe SDB according to BMI is shown in figure 6. In accordance with increases in BMI, percentages of high acti-CT90 increased among those with moderate-to-severe SDB. This tendency was not evident among those without SDB and with mild SDB (supplementary figures S10 and S11, respectively). 


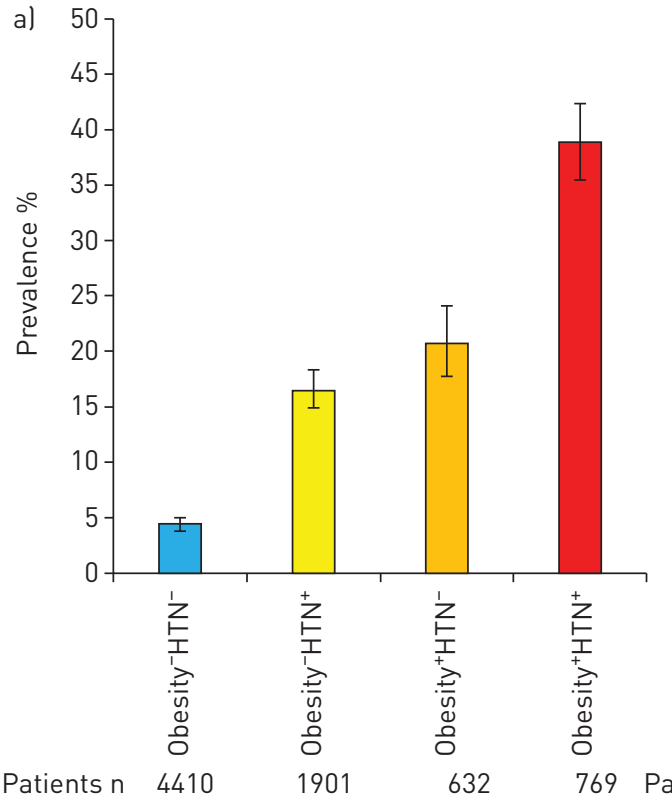

b)

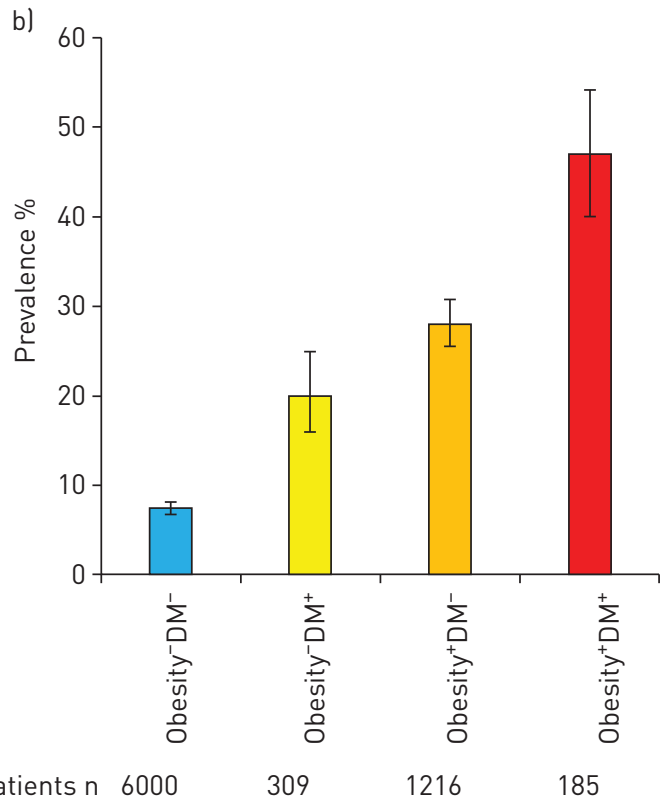

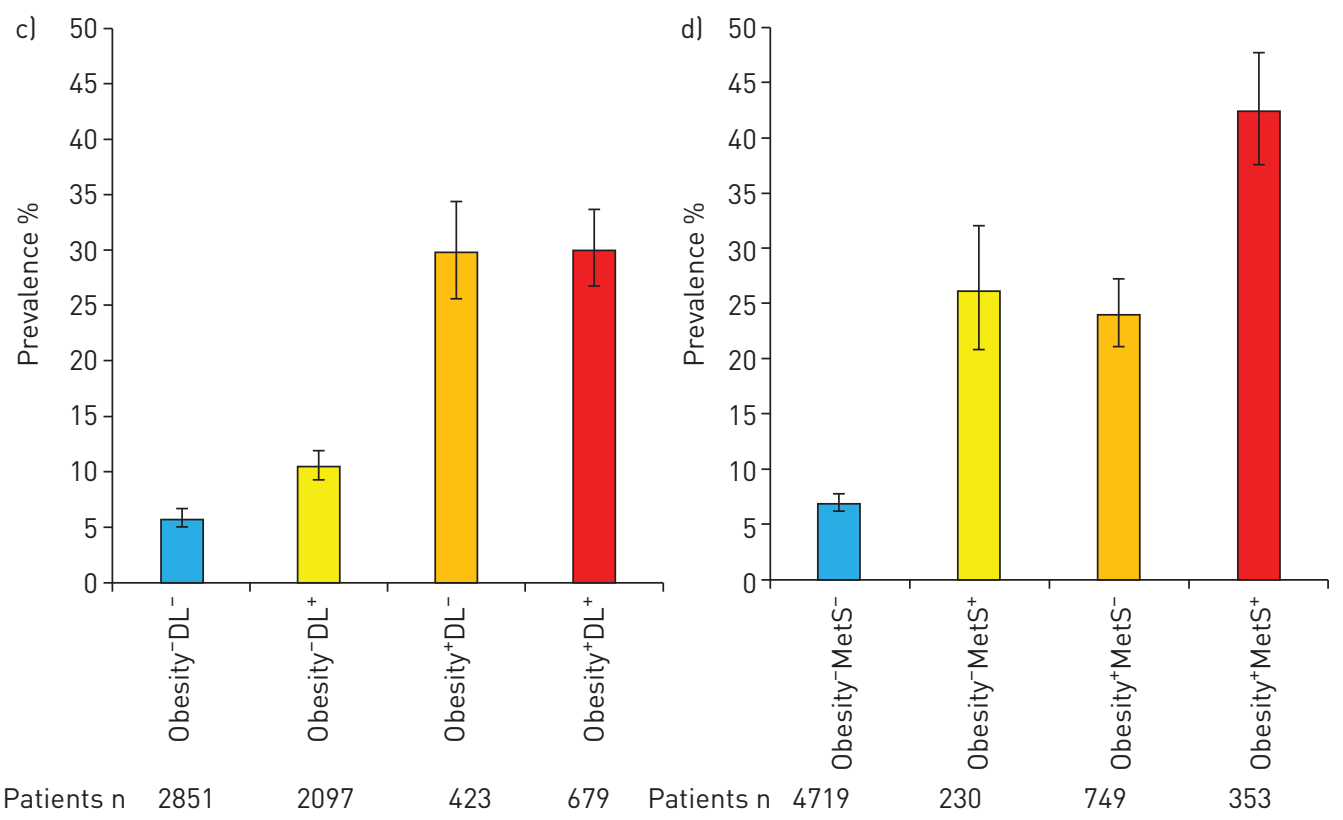

FIGURE 4 Percentages of moderate-to-severe sleep disordered breathing according to comorbidity and/or obesity. a) Presence or absence of hypertension (HTN) and/or obesity; b) presence or absence of diabetes (DM) and/or obesity; c) presence or absence of dyslipidaemia (DL) and/or obesity; d) presence or absence of metabolic syndrome (MetS) and/or obesity. The number below each bar graph represents the sample size of that group. Data are presented as point estimates $(95 \% \mathrm{CI})$. Data on DL and MetS were limited to participants in a fasting state.

\section{Discussion}

In terms of sex and menopausal status, the current study shows notable epidemiological evidence about the contribution of obesity and comorbidities to SDB. The prevalence of SDB in East Asians was $41.0 \%$ (95\% CI 39.9-42.1\%), 46.9\% (45.8-48.0\%), 10.1\% (9.5-10.8\%) and 2.0\% (1.7-2.3\%) in normal, mild, moderate and severe SDB, respectively, with notable sex differences evident (males >post-menopausal females $>$ premenopausal females). These results did not differ greatly from other studies in Caucasian or Hispanic/Latino participants. BMI, waist circumference and body fat percentage were contributors to SDB, but the degrees of the contributions were different by sex. Premenopausal females were the most susceptible to obesity in the context of SDB prevalence. Comorbidities such as hypertension, diabetes and metabolic syndrome with and without obesity were independently associated with SDB, and their 

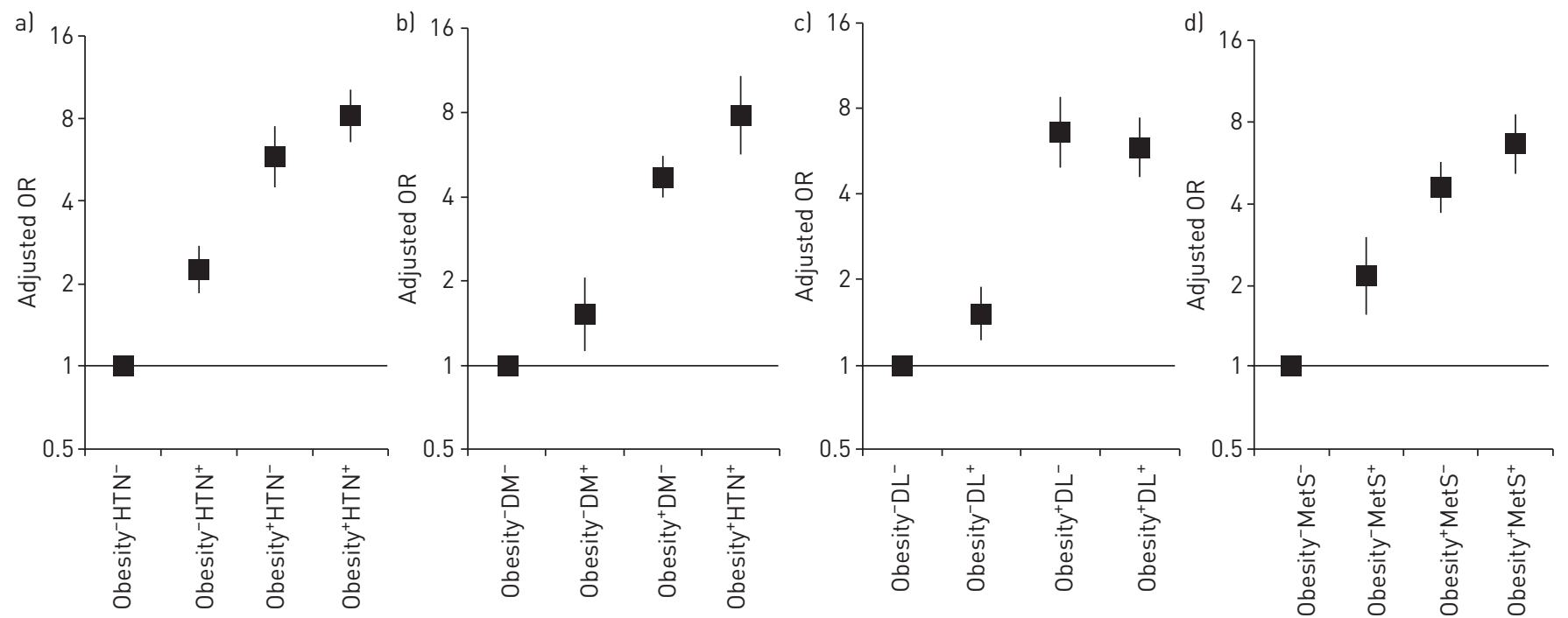

FIGURE 5 Odds ratios for moderate-to-severe sleep disordered breathing according to comorbidity and/or obesity. a) Presence or absence of hypertension (HTN) and/or obesity; b) presence or absence of diabetes (DM) and/or obesity; c) presence or absence of dyslipidaemia (DL) and/or obesity; d) presence or absence of metabolic syndrome (MetS) and/or obesity. Data are presented as point estimates (95\% CI). Vertical lines are shown as the log-transformed scale. The results were adjusted for age, sex, smoking and alcohol status. Data on DL and MetS were limited to participants in a fasting state.

coincidence increased the probability of SDB. As to hypertension, the number of antihypertensive drugs was associated with SDB. However, dyslipidaemia in addition to obesity was not additively associated with SDB. Percentages of high acti-CT90 increased among those with moderate-to-severe SDB according to increases in obesity.

In this study, we showed the percentages of SDB in the presence or absence of hypertension and diabetes according to sex and menopausal status in relation to BMI (figure 2). Previously, SDB has been reported to be associated with various comorbidities such as hypertension [8], diabetes [9, 10], dyslipidaemia [11, $12]$ and metabolic syndrome $[13,14]$. However, the reverse viewpoint has hardly been discussed, although

FIGURE 6 Percentages of high actigraphy-modified cumulative percentage of sleep time with peripheral oxygen saturation $<90 \%$ (acti-CT90) among moderate-tosevere sleep disordered breathing (SDB) patients according to severity of obesity. Data are presented as point estimates $(95 \% \mathrm{CI})$. BMI: body mass index.

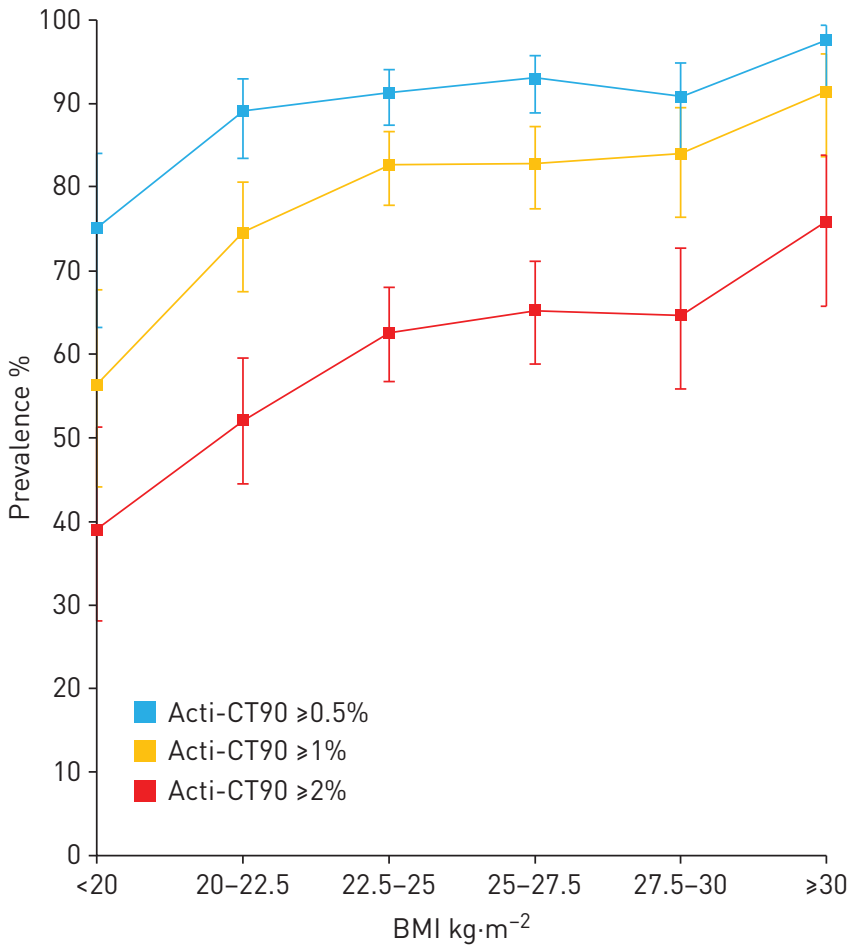


the direction of the causal relationship would be from SDB to comorbidities [18]. Not surprisingly, a positive association was observed, but we showed the real-world percentages of SDB for each comorbidity examined, especially according to sex and menopausal status groupings and BMI values. To our knowledge, this is the first study to evaluate these specific aspects. Clinically, real-world data on SDB for various comorbidity and obesity groupings are of practical value and would be helpful in formulating public health policies. In particular, the number of antihypertensive drugs was associated with SDB (figure 3). When a number of antihypertensive drugs are required, the degree of hypertension would be severe, which could explain these results on the prevalence of SDB. Questioning a patient on the number of antihypertensive drugs is very simple and would be helpful for detecting SDB in daily clinical settings. The percentage of moderate-to-severe SDB in those with hypertension was previously reported to be $\sim 30 \%$ [28]. In this study, the result was $22.9 \%$. In addition, the percentage of moderate-to-severe SDB in those with resistant hypertension was reported to be as high as $80 \%$ [15]. However, it was $39.1 \%$ in our study. This discrepancy was probably due to characteristics of the participants; that is, participants in this study were from a general population and were East Asian. Therefore, in a general population, the percentage might not be as high as previously reported; however, educational efforts on this issue are important because the result of $40 \%$ is sufficiently high to warrant attention.

In examining the impact of combinations of comorbidities and obesity on SDB (figures 4 and 5) the impact was higher in the order of doubly negative, only comorbidity present, only obesity present and doubly positive. Therefore, a comorbidity without obesity was a risk factor for SDB, although it was reported that obesity was a strong confounding factor between SDB and metabolic comorbidities [29]. In particular, premenopausal females were more affected by obesity than the other groups, indicating the need for treating obesity in that group as control of body weight has been shown to be important [30]. In addition, the predictive value of clinical symptoms such as EDS or poor sleep quality was slight, if any, and comorbidities or obesity had a greater impact on the presence of SDB, which may reinforce the importance of identifying metabolic comorbidities.

The association of dyslipidaemia was not additive to obesity in relation to SDB, differing from the other comorbidities examined. There have been reports that dyslipidaemia is not associated with SDB [29, 31]. Conversely, CPAP therapy was reported to reduce postprandial lipidaemia [32], while weight loss rather than CPAP therapy was more effective in improving dyslipidaemia [30]. Therefore, the impact of obesity may be strong for dyslipidaemia compared to other metabolic comorbidities.

Although many studies have shown an association between obesity and SDB [3,17], there were few reports of differences between obesity parameters [33]. In this study, we firstly presented the comprehensive association between obesity parameters and SDB in a large community cohort according to sex differences and menopausal status $(n=7713)$. Not surprisingly, the more severe the obesity parameters were, the more SDB participants were detected; and each obesity parameter was independently associated with SDB (supplementary table S2). However, these three parameters were well correlated with each other (supplementary table S1). Therefore, it might be better to use only one of them in a population-based study for fear of collinearity. In addition, associations between SDB and obesity parameters substituted by odds ratios were different between groups (post-menopausal females $<$ males $<$ premenopausal females). A strong impact of obesity on premenopausal females was suggested previously [34]. Therefore, among the groups examined, premenopausal females may be the most susceptible to obesity in the context of SDB development.

Previously it was shown that those who met the cut-off BMI of $32.3 \mathrm{~kg} \cdot \mathrm{m}^{-2}$ had a six-fold increase in the prevalence of moderate SDB in the United States [35]. However, in this study, the cut-off of BMI $25 \mathrm{~kg} \cdot \mathrm{m}^{-2}$ was the point at which the prevalence of SDB was increased. This value was much lower than previously reported. BMI $\geqslant 25 \mathrm{~kg} \cdot \mathrm{m}^{-2}$ is considered as obesity in Japan (as defined by the Japan Society for the Study of Obesity). Although obesity is defined by BMI $\geqslant 30 \mathrm{~kg} \cdot \mathrm{m}^{-2}$ in Westerners, only $4 \%$ of Japanese people meet this criterion, while $\sim 30 \%$ of males and $\sim 20 \%$ of females have BMI $\geqslant 25 \mathrm{~kg} \cdot \mathrm{m}^{-2}$ [36]. Therefore, in East Asians, we may need to pay attention to the presence of moderate-to-severe SDB at lower BMIs than among Westerners [37].

Lastly, we showed a specific association between intermittent and continuous hypoxia according severity of obesity (figure 6, supplementary figures S10 and S11). When obesity developed, continuous hypoxia accordingly deteriorated even in moderate-to-severe intermittent hypoxia (acti-ODI3\% $\geqslant 15$ events $\cdot \mathrm{h}^{-1}$ ). Intermittent hypoxia was reported to enhance sympathetic responses to continuous hypoxia [38], so the double negative effects may contribute to a poor prognosis, especially in obese patients with moderate-to-severe SDB [39].

Several limitations should be mentioned. First, as this study was cross-sectional, it remains unresolved about causality. Also, as data were from only East Asian participants, the results of this study might not be 
generalised to other ethnic groups [37]. Nonetheless, these are the first such results from a large East Asian cohort, and these findings would be valuable in assessing worldwide data. Second, SDB was evaluated by pulse oximetry. Previous studies using simple ODI without adjusting for objective sleep duration had drawbacks in the diagnosis of SDB. Indeed, it is better to use polysomnography for diagnosing SDB. However, polysomnography is burdensome, requiring much effort. In this study, as ODI was adjusted for objective sleep duration by actigraphy, which is similar to sleep duration by polysomnography, this acti-ODI might not deviate from information obtained with polysomnography as shown in the methods section. Third, a certain number of participants were nonfasting, and nonfasting participants were excluded from the analysis of dyslipidaemia and metabolic syndrome, so these participants differed from those included in the analysis of hypertension and diabetes. However, random selection of participants with a fasting state would minimise the bias. The large number of those with a fasting state $(n=6051)$ would be sufficient for statistical power.

In conclusion, metabolic comorbidities such as hypertension, diabetes, dyslipidaemia, metabolic syndrome and obesity were independently associated with SDB, and their coincidence increased the probability of SDB. However, dyslipidaemia in addition to obesity was not additively associated with SDB. Metabolic comorbidities as well as obesity should be given attention in terms of the presence of SDB.

Acknowledgements: We are grateful to Nagahama City Office and the nonprofit organisation Zeroji Club for their assistance in performing the Nagahama study. We are grateful to Yoshiro Toyama, Masanori Azuma, Ryo Tachikawa and Morito Inouchi (Kyoto University Graduate School of Medicine, Kyoto, Japan) for their assistance in collecting data. We are also grateful to Shunsuke Nagashima, Chiaki Tojo and Makoto Yamashita (Kyoto University Graduate School of Medicine) for their assistance in analysing the actigraphy data.

Author contributions: Study design: T. Matsumoto and K. Chin; data collection: T. Matsumoto, K. Murase, Y. Tabara, T. Minami, O. Kanai, H. Takeyama, N. Takahashi, S. Hamada, K. Tanizawa, T. Wakamura, N. Komenami, K. Setoh, T. Kawaguchi, T. Tsutsumi, Y. Takahashi, T. Nakayama, T. Hirai, F. Matsuda and K. Chin; data analysis and interpretation: T. Matsumoto and K. Chin; manuscript drafting: T. Matsumoto and K. Chin; critical revision: K. Murase, Y. Tabara, T. Minami, O. Kanai, H. Takeyama, N. Takahashi, S. Hamada, K. Tanizawa, T. Wakamura, N. Komenami, K. Setoh, T. Kawaguchi, T. Tsutsumi, S. Morita, Y. Takahashi, T. Nakayama, T. Hirai and F. Matsuda; approval of the final version of manuscript: T. Matsumoto, K. Murase, Y. Tabara, T. Minami, O. Kanai, H. Takeyama, N. Takahashi, S. Hamada, K. Tanizawa, T. Wakamura, N. Komenami, K. Setoh, T. Kawaguchi, T. Tsutsumi, S. Morita, Y. Takahashi, T. Nakayama, T. Hirai, F. Matsuda and K. Chin.

Conflict of interest: T. Matsumoto has nothing to disclose. K. Murase reports grants from Philips-Respironics, Teijin Pharma, Fukuda Denshi, Fukuda Lifetec Keiji, ResMed and Japan Society for the Promotion of Science, outside the submitted work. Y. Tabara reports grants from Japan Agency for Medical Research and Development (AMED) and The Ministry of Education, Culture, Sports, Science and Technology in Japan, during the conduct of the study. T. Minami reports personal fees from Teijin Zaitakuiryou, outside the submitted work. O. Kanai has nothing to disclose. H. Takeyama reports grants from Philips-Respironics, ResMed, Fukuda Denshi, Fukuda Lifetec Keiji and Teijin Pharma, outside the submitted work. N. Takahashi reports grants from Philips-Respironics, ResMed, Fukuda Denshi and Fukuda Lifetec Keiji, outside the submitted work. S. Hamada reports grants from Teijin Pharma, outside the submitted work. $\mathrm{K}$. Tanizawa has nothing to disclose. T. Wakamura has nothing to disclose. N. Komenami has nothing to disclose. K. Setoh has nothing to disclose. T. Kawaguchi has nothing to disclose. T. Tsutsumi has nothing to disclose. S. Morita has nothing to disclose. Y. Takahashi has nothing to disclose. T. Nakayama has nothing to disclose. T. Hirai has nothing to disclose. F. Matsuda reports grants from Kyoto University, the Ministry of Education, Culture, Sports, Science and Technology in Japan, Japan Agency for Medical Research and Development (AMED) and The Takeda Medical Research Foundation, during the conduct of the study. K. Chin reports grants from the Japanese Ministry of Education, Culture, Sports, Science and Technology, grants from the Intractable Respiratory Diseases and Pulmonary Hypertension Research Group, the Ministry of Health, Labour and Welfare, Japan, grants from the Research Foundation for Healthy Aging, grants from Health, Labour and Welfare Sciences Research Grants, Research on Region Medical, grants from the Center of Innovation Program, and the Global University Project from Japan Science and Technology Agency, Japan Agency for Medical Research and Development, during the conduct of the study; grants and personal fees from Philips-Respironics, Teijin Pharma, Fukuda Denshi, Fukuda Lifetec Keiji, GlaxoSmithKline and Resmed, grants from KYORIN Pharmaceutical Co., Ltd and Nippon Boehringer Ingelheim Co., Ltd, personal fees from MSD, Astellas Pharma and Eisai Co., Ltd, outside the submitted work.

Support statement: This study was funded by a University Grant, a Grant-in-Aid for Scientific Research from the Ministry of Education, Culture, Sports, Science and Technology in Japan (25293141, 26670313, 26293198, 17H04182, 17H04126, 17H04123, 18K18450), the Center of Innovation Program, and the Global University Project from Japan Science and Technology Agency, Japan Agency for Medical Research and Development (AMED) under grant number dk0207006, dk0207027, ek0109070, ek0109283, ek0109196, ek0109348, kk0205008, ek0210066, ek0210096, ek0210116, and le0110005, grants from the Japanese Ministry of Education, Culture, Sports, Science and Technology, the Intractable Respiratory Diseases and Pulmonary Hypertension Research Group from the Ministry of Health, Labour and Welfare of Japan (H29-intractable diseases-general-027), the Takeda Medical Research Foundation, Mitsubishi Foundation, Daiwa Securities Health Foundation, Sumitomo Foundation, the Research Foundation for Healthy Aging, and Health, Labour and Welfare Sciences Research Grants, Research on Region Medical (H28-iryo-ippan-016, H30-iryo-ippan-009). The Dept of Respiratory Care and Sleep Control Medicine is funded by Philips-Respironics, Fukuda Denshi, Fukuda Lifetec Keiji, and ResMed to Kyoto University. The Dept of Advanced Medicine for Respiratory Failure is a Dept of Collaborative Research Laboratory funded by Teijin Pharma. Funding information for this article has been deposited with the Crossref Funder Registry. 


\section{References}

1 Cefalu WT, Bray GA, Home PD, et al. Advances in the science, treatment, and prevention of the disease of obesity: reflections from a diabetes care editors' expert forum. Diabetes Care 2015; 38: 1567-1582.

2 Rahmouni K. Obesity-associated hypertension: recent progress in deciphering the pathogenesis. Hypertension 2014; 64: 215-221

3 Jordan AS, McSharry DG, Malhotra A. Adult obstructive sleep apnoea. Lancet 2014; 383: 736-747.

4 Heinzer R, Vat S, Marques-Vidal P, et al. Prevalence of sleep-disordered breathing in the general population: the HypnoLaus study. Lancet Respir Med 2015; 3: 310-318.

5 Matsumoto T, Murase K, Tabara Y, et al. Impact of sleep characteristics and obesity on diabetes and hypertension across genders and menopausal status: the Nagahama study. Sleep 2018; 41: zsy071.

6 Namen AM, Chatterjee A, Huang KE, et al. Recognition of sleep apnea is increasing. Analysis of trends in two large, representative databases of outpatient practice. Ann Am Thorac Soc 2016; 13: 2027-2034.

7 Peppard PE, Young T, Barnet $\mathrm{JH}$, et al. Increased prevalence of sleep-disordered breathing in adults. Am Epidemiol 2013; 177: 1006-1014.

8 Peppard PE, Young T, Palta M, et al. Prospective study of the association between sleep-disordered breathing and hypertension. N Engl J Med 2000; 342: 1378-1384.

9 Aronsohn RS, Whitmore H, Van Cauter E, et al. Impact of untreated obstructive sleep apnea on glucose control in type 2 diabetes. Am J Respir Crit Care Med 2010; 181: 507-513.

10 Kendzerska T, Gershon AS, Hawker G, et al. Obstructive sleep apnea and incident diabetes. A historical cohort study. Am J Respir Crit Care Med 2014; 190: 218-225.

11 Toyama Y, Chin K, Chihara Y, et al. Association between sleep apnea, sleep duration, and serum lipid profile in an urban, male, working population in Japan. Chest 2013; 143: 720-728.

12 Wu WT, Tsai SS, Shih TS, et al. The association between obstructive sleep apnea and metabolic markers and lipid profiles. PLoS One 2015; 10: e0130279.

13 Chin K, Oga T, Takahashi K, et al. Associations between obstructive sleep apnea, metabolic syndrome, and sleep duration, as measured with an actigraph, in an urban male working population in Japan. Sleep 2010; 33: 89-95.

14 Akahoshi T, Uematsu A, Akashiba T, et al. Obstructive sleep apnoea is associated with risk factors comprising the metabolic syndrome. Respirology 2010; 15: 1122-1126.

15 Logan AG, Perlikowski SM, Mente A, et al. High prevalence of unrecognized sleep apnoea in drug-resistant hypertension. J Hypertens 2001; 19: 2271-2277.

16 Lam DC, Lui MM, Lam JC, et al. Prevalence and recognition of obstructive sleep apnea in Chinese patients with type 2 diabetes mellitus. Chest 2010; 138: 1101-1107.

17 Pillar G, Shehadeh N. Abdominal fat and sleep apnea: the chicken or the egg? Diabetes Care 2008; 31: Suppl. 2, S303-S309.

18 Arnardottir ES, Mackiewicz M, Gislason T, et al. Molecular signatures of obstructive sleep apnea in adults: a review and perspective. Sleep 2009; 32: 447-470.

19 Azarbarzin A, Sands SA, Stone KL, et al. The hypoxic burden of sleep apnoea predicts cardiovascular disease-related mortality: the Osteoporotic Fractures in Men Study and the Sleep Heart Health Study. Eur Heart $J$ 2019; 40: 1149-1157.

20 Young T, Finn L, Austin D, et al. Menopausal status and sleep-disordered breathing in the Wisconsin Sleep Cohort Study. Am J Respir Crit Care Med 2003; 167: 1181-1185.

21 Bixler EO, Vgontzas AN, Lin HM, et al. Prevalence of sleep-disordered breathing in women: effects of gender. Am J Respir Crit Care Med 2001; 163: 608-613.

22 Flegal KM, Kit BK, Orpana H, et al. Association of all-cause mortality with overweight and obesity using standard body mass index categories: a systematic review and meta-analysis. JAMA 2013; 309: 71-82.

23 Lean MJ, Han TS. Waist worries. Am J Clin Nutr 2002; 76: 699-700.

24 Roubenoff R. Applications of bioelectrical impedance analysis for body composition to epidemiologic studies. Am J Clin Nutr 1996; 64: 3 Suppl., 459S-462S

25 Matsuzawa Y. Metabolic syndrome - definition and diagnostic criteria in Japan. J Atheroscler Thromb 2005; 12: 301.

26 Arnardottir ES, Bjornsdottir E, Olafsdottir KA, et al. Obstructive sleep apnoea in the general population: highly prevalent but minimal symptoms. Eur Respir J 2016; 47: 194-202.

27 Redline S, Sotres-Alvarez D, Loredo J, et al. Sleep-disordered breathing in Hispanic/Latino individuals of diverse backgrounds. The Hispanic Community Health Study/Study of Latinos. Am J Respir Crit Care Med 2014; 189: 335-344.

28 Somers VK, White DP, Amin R, et al. Sleep apnea and cardiovascular disease: an American Heart Association/ American College Of Cardiology Foundation Scientific Statement from the American Heart Association Council for High Blood Pressure Research Professional Education Committee, Council on Clinical Cardiology, Stroke Council, and Council On Cardiovascular Nursing. In collaboration with the National Heart, Lung, and Blood Institute National Center on Sleep Disorders Research (National Institutes of Health). Circulation 2008; 118: $1080-1111$.

29 Wakabayashi Y, Oka R, Nakaya M, et al. Associations between sleep-disordered breathing and metabolic risk factors beyond obesity. J Diabetes Res 2018; 2018: 1567683.

30 Chirinos JA, Gurubhagavatula I, Teff K, et al. CPAP, weight loss, or both for obstructive sleep apnea. $N$ Engl $J$ Med 2014; 370: 2265-2275.

31 Ozol D, Turkay C, Kasapoglu B, et al. Relationship between components of metabolic syndrome and polysomnographic findings in obstructive sleep apnea. Metab Syndr Relat Disord 2011; 9: 13-18.

32 Phillips CL, Yee BJ, Marshall NS, et al. Continuous positive airway pressure reduces postprandial lipidemia in obstructive sleep apnea: a randomized, placebo-controlled crossover trial. Am J Respir Crit Care Med 2011; 184: 355-361.

33 Oğretmenoğlu O, Süslü AE, Yücel OT, et al. Body fat composition: a predictive factor for obstructive sleep apnea. Laryngoscope 2005; 115: 1493-1498.

34 Ross R, Freeman J, Hudson R, et al. Abdominal obesity, muscle composition, and insulin resistance in premenopausal women. J Clin Endocrinol Metab 2002; 87: 5044-5051. 
Young T, Peppard PE, Taheri S. Excess weight and sleep-disordered breathing. J Appl Physiol 2005; 99: 1592-1599. Japanese Ministry of Health, Labour and Welfare. National Health and Nutrition Survey. Date last accessed: September 16, 2019. www.mhlw.go.jp/stf/seisakunitsuite/bunya/kenkou_iryou/kenkou/eiyou/h29-houkoku.html.

37 Chen X, Wang R, Lutsey PL, et al. Racial/ethnic differences in the associations between obesity measures and severity of sleep-disordered breathing: the Multi-Ethnic Study of Atherosclerosis. Sleep Med 2016; 26: 46-53.

38 Leuenberger UA, Hogeman CS, Quraishi SA, et al. Short-term intermittent hypoxia enhances sympathetic responses to continuous hypoxia in humans. J Appl Physiol 2007; 103: 835-842.

39 Drager LF, Togeiro SM, Polotsky VY, et al. Obstructive sleep apnea: a cardiometabolic risk in obesity and the metabolic syndrome. J Am Coll Cardiol 2013; 62: 569-576. 